\title{
Some Properties of Some Special Matrices. Part II
}

\author{
Xiaopeng Yue \\ Qingdao University of Science \\ and Technology \\ China
}

\author{
Dahai $\mathrm{Hu}$ \\ Qingdao University of Science \\ and Technology \\ China
}

\author{
Xiquan Liang \\ Qingdao University of Science \\ and Technology \\ China
}

Summary. This article provides definitions of idempotent, nilpotent, involutory, self-reversible, similar, and congruent matrices, the trace of a matrix and their main properties.

MML identifier: MATRIX_8, version: 7.6.01 4.53.937

The terminology and notation used here are introduced in the following articles: [7], [3], [1], [9], [8], [6], [4], [2], [5], [11], and [10].

We adopt the following convention: $n$ is a natural number, $K$ is a field, and $M_{1}, M_{2}, M_{3}, M_{4}, M_{5}, M_{6}$ are matrices over $K$ of dimension $n$.

Let $n$ be a natural number, let $K$ be a field, and let $M_{1}$ be a matrix over $K$ of dimension $n$. We say that $M_{1}$ is idempotent if and only if:

(Def. 1) $\quad M_{1} \cdot M_{1}=M_{1}$.

We say that $M_{1}$ is 2-nilpotent if and only if:

(Def. 2) $\quad M_{1} \cdot M_{1}=\left(\begin{array}{ccc}0 & \ldots & 0 \\ \vdots & \ddots & \vdots \\ 0 & \ldots & 0\end{array}\right)_{K}^{n \times n}$.

We say that $M_{1}$ is involutory if and only if: 
(Def. 3) $M_{1} \cdot M_{1}=\left(\begin{array}{ccc}1 & & 0 \\ & \ddots & \\ 0 & & 1\end{array}\right)_{K}^{n \times n}$.

We say that $M_{1}$ is self invertible if and only if:

(Def. 4) $\quad M_{1}$ is invertible and $M_{1} \smile=M_{1}$.

We now state a number of propositions:

(1) $\left(\begin{array}{lll}1 & & 0 \\ & \ddots & \\ 0 & & 1\end{array}\right)_{K}^{n \times n}$ is idempotent and involutory.

(2) If $n>0$, then $\left(\begin{array}{ccc}0 & \ldots & 0 \\ \vdots & \ddots & \vdots \\ 0 & \ldots & 0\end{array}\right)_{K}^{n \times n}$ is idempotent and 2-nilpotent.

(3) If $n>0$ and $M_{2}=M_{1}^{\mathrm{T}}$, then $M_{1}$ is idempotent iff $M_{2}$ is idempotent.

(4) If $M_{1}$ is involutory, then $M_{1}$ is invertible.

(5) If $M_{1}$ is idempotent and $M_{2}$ is idempotent and $M_{1}$ is permutable with $M_{2}$, then $M_{1} \cdot M_{1}$ is permutable with $M_{2} \cdot M_{2}$.

(6) If $n>0$ and $M_{1}$ is idempotent and $M_{2}$ is idempotent and $M_{1}$ is permutable with $M_{2}$ and $M_{1} \cdot M_{2}=\left(\begin{array}{ccc}0 & \ldots & 0 \\ \vdots & \ddots & \vdots \\ 0 & \ldots & 0\end{array}\right)_{K}^{n \times n}$, then $M_{1}+M_{2}$ is idempotent.

(7) If $n>0$ and $M_{1}$ is idempotent and $M_{2}$ is idempotent and $M_{1} \cdot M_{2}=$ $-M_{2} \cdot M_{1}$, then $M_{1}+M_{2}$ is idempotent.

(8) If $M_{1}$ is idempotent and $M_{2}$ is invertible, then $M_{2} \smile \cdot M_{1} \cdot M_{2}$ is idempotent.

(9) If $n>0$ and $M_{1}$ is invertible and idempotent, then $M_{1} \smile$ is idempotent.

(10) If $M_{1}$ is invertible and idempotent, then $M_{1}=\left(\begin{array}{ccc}1 & & 0 \\ & \ddots & \\ 0 & & 1\end{array}\right)_{K}^{n \times n}$.

(11) If $M_{1}$ is idempotent and $M_{2}$ is idempotent and $M_{1}$ is permutable with $M_{2}$, then $M_{1} \cdot M_{2}$ is idempotent.

(12) If $n>0$ and $M_{1}$ is idempotent and $M_{2}$ is idempotent and $M_{1}$ is permutable with $M_{2}$ and $M_{3}=M_{1}^{\mathrm{T}} \cdot M_{2}^{\mathrm{T}}$, then $M_{3}$ is idempotent.

(13) If $M_{1}$ is idempotent and $M_{2}$ is idempotent and $M_{1}$ is invertible, then $M_{1} \cdot M_{2}$ is idempotent.

(14) If $n>0$ and $M_{1}$ is idempotent and orthogonal, then $M_{1}$ is symmetrical. 
(15) If $M_{1}$ is idempotent and $M_{2}$ is idempotent and $M_{2} \cdot M_{1}=$ $\left(\begin{array}{ccc}1 & & 0 \\ & \ddots & \\ 0 & & 1\end{array}\right)_{K}^{n \times n}$, then $M_{1} \cdot M_{2}$ is idempotent.

(16) If $M_{1}$ is idempotent and orthogonal, then $M_{1}=\left(\begin{array}{ccc}1 & & 0 \\ & \ddots & \\ 0 & & 1\end{array}\right)_{K}^{n \times n}$.

(17) If $n>0$ and $M_{1}$ is symmetrical and $M_{2}=M_{1}^{\mathrm{T}}$, then $M_{1} \cdot M_{2}$ is symmetrical.

(18) If $n>0$ and $M_{1}$ is symmetrical and $M_{2}=M_{1}^{\mathrm{T}}$, then $M_{2} \cdot M_{1}$ is symmetrical.

(19) If $M_{1}$ is invertible and $M_{1} \cdot M_{2}=M_{1} \cdot M_{3}$, then $M_{2}=M_{3}$.

(20) If $M_{1}$ is invertible and $M_{2} \cdot M_{1}=M_{3} \cdot M_{1}$, then $M_{2}=M_{3}$.

(21) If $n>0$ and $M_{1}$ is invertible and $M_{2} \cdot M_{1}=\left(\begin{array}{ccc}0 & \ldots & 0 \\ \vdots & \ddots & \vdots \\ 0 & \ldots & 0\end{array}\right)_{K}^{n \times n}$, then $M_{2}=\left(\begin{array}{ccc}0 & \ldots & 0 \\ \vdots & \ddots & \vdots \\ 0 & \ldots & 0\end{array}\right)_{K}^{n \times n}$

(22) If $n>0$ and $M_{1}$ is invertible and $M_{2} \cdot M_{1}=\left(\begin{array}{ccc}0 & \ldots & 0 \\ \vdots & \ddots & \vdots \\ 0 & \ldots & 0\end{array}\right)_{K}^{n \times n}$, then $M_{2}=\left(\begin{array}{ccc}0 & \ldots & 0 \\ \vdots & \ddots & \vdots \\ 0 & \ldots & 0\end{array}\right)_{K}^{n \times n}$

(23) If $M_{1}$ is 2-nilpotent and permutable with $M_{2}$ and $n>0$, then $M_{1} \cdot M_{2}$ is 2-nilpotent.

(24) If $n>0$ and $M_{1}$ is 2-nilpotent and $M_{2}$ is 2-nilpotent and $M_{1}$ is permutable with $M_{2}$ and $M_{1} \cdot M_{2}=\left(\begin{array}{ccc}0 & \ldots & 0 \\ \vdots & \ddots & \vdots \\ 0 & \ldots & 0\end{array}\right)_{K}^{n \times n}$, then $M_{1}+M_{2}$ is 2-nilpotent.

(25) If $M_{1}$ is 2-nilpotent and $M_{2}$ is 2-nilpotent and $M_{1} \cdot M_{2}=-M_{2} \cdot M_{1}$ and $n>0$, then $M_{1}+M_{2}$ is 2-nilpotent.

(26) If $M_{1}$ is 2-nilpotent and $M_{2}=M_{1}^{\mathrm{T}}$ and $n>0$, then $M_{2}$ is 2-nilpotent. 
(27) If $M_{1}$ is 2-nilpotent and idempotent, then $M_{1}=\left(\begin{array}{ccc}0 & \ldots & 0 \\ \vdots & \ddots & \vdots \\ 0 & \ldots & 0\end{array}\right)_{K}^{n \times n}$.

(28) If $n>0$, then $\left(\begin{array}{ccc}0 & \ldots & 0 \\ \vdots & \ddots & \vdots \\ 0 & \ldots & 0\end{array}\right)_{K}^{n \times n} \neq\left(\begin{array}{ccc}1 & & 0 \\ & \ddots & \\ 0 & & 1\end{array}\right)_{K}^{n \times n}$.

(29) If $n>0$ and $M_{1}$ is 2-nilpotent, then $M_{1}$ is not invertible.

(30) If $M_{1}$ is self invertible, then $M_{1}$ is involutory.

(31) $\left(\begin{array}{lll}1 & & 0 \\ & \ddots & \\ 0 & & 1\end{array}\right)_{K}^{n \times n}$ is self invertible.

(32) If $M_{1}$ is self invertible and idempotent, then $M_{1}=\left(\begin{array}{ccc}1 & & 0 \\ & \ddots & \\ 0 & & 1\end{array}\right)_{K}^{n \times n}$.

(33) If $M_{1}$ is self invertible and symmetrical, then $M_{1}$ is orthogonal.

Let $n$ be a natural number, let $K$ be a field, and let $M_{1}, M_{2}$ be matrices over $K$ of dimension $n$. We say that $M_{1}$ is similar to $M_{2}$ if and only if:

(Def. 5) There exists a matrix $M$ over $K$ of dimension $n$ such that $M$ is invertible and $M_{1}=M^{\smile} \cdot M_{2} \cdot M$.

Let us notice that the predicate $M_{1}$ is similar to $M_{2}$ is reflexive and symmetric.

The following propositions are true:

(34) If $M_{1}$ is similar to $M_{2}$ and $M_{2}$ is similar to $M_{3}$ and $n>0$, then $M_{1}$ is similar to $M_{3}$.

(35) If $M_{1}$ is similar to $M_{2}$ and $M_{2}$ is idempotent, then $M_{1}$ is idempotent.

(36) If $M_{1}$ is similar to $M_{2}$ and $M_{2}$ is 2-nilpotent and $n>0$, then $M_{1}$ is 2-nilpotent.

(37) If $M_{1}$ is similar to $M_{2}$ and $M_{2}$ is involutory, then $M_{1}$ is involutory.

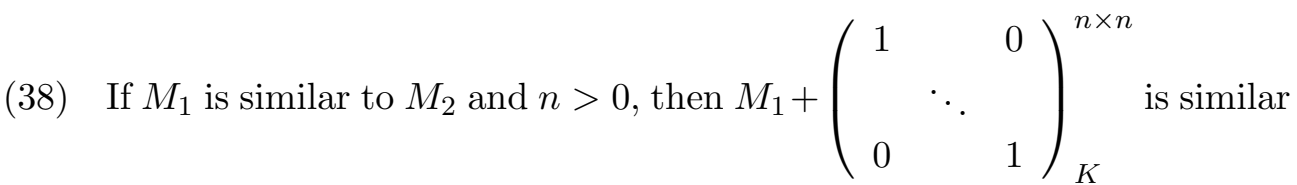
to $M_{2}+\left(\begin{array}{ccc}1 & & 0 \\ & \ddots & \\ 0 & & 1\end{array}\right)_{K}^{n \times n}$.

(39) If $M_{1}$ is similar to $M_{2}$ and $n>0$, then $M_{1}+M_{1}$ is similar to $M_{2}+M_{2}$.

(40) If $M_{1}$ is similar to $M_{2}$ and $n>0$, then $M_{1}+M_{1}+M_{1}$ is similar to $M_{2}+M_{2}+M_{2}$. 
(41) If $M_{1}$ is invertible, then $M_{2} \cdot M_{1}$ is similar to $M_{1} \cdot M_{2}$.

(42) If $M_{2}$ is invertible and $M_{1}$ is similar to $M_{2}$ and $n>0$, then $M_{1}$ is invertible.

(43) If $M_{2}$ is invertible and $M_{1}$ is similar to $M_{2}$ and $n>0$, then $M_{1}{ }^{\smile}$ is similar to $M_{2} \smile$.

Let $n$ be a natural number, let $K$ be a field, and let $M_{1}, M_{2}$ be matrices over $K$ of dimension $n$. We say that $M_{1}$ is congruent to $M_{2}$ if and only if:

(Def. 6) There exists a matrix $M$ over $K$ of dimension $n$ such that $M$ is invertible and $M_{1}=M^{\mathrm{T}} \cdot M_{2} \cdot M$.

Next we state several propositions:

(44) If $n>0$, then $M_{1}$ is congruent to $M_{1}$.

(45) If $M_{1}$ is congruent to $M_{2}$ and $n>0$, then $M_{2}$ is congruent to $M_{1}$.

(46) If $M_{1}$ is congruent to $M_{2}$ and $M_{2}$ is congruent to $M_{3}$ and $n>0$, then $M_{1}$ is congruent to $M_{3}$.

(47) If $M_{1}$ is congruent to $M_{2}$ and $n>0$, then $M_{1}+M_{1}$ is congruent to $M_{2}+M_{2}$.

(48) If $M_{1}$ is congruent to $M_{2}$ and $n>0$, then $M_{1}+M_{1}+M_{1}$ is congruent to $M_{2}+M_{2}+M_{2}$.

(49) If $M_{1}$ is orthogonal, then $M_{2} \cdot M_{1}$ is congruent to $M_{1} \cdot M_{2}$.

(50) If $M_{2}$ is invertible and $M_{1}$ is congruent to $M_{2}$ and $n>0$, then $M_{1}$ is invertible.

(51) If $M_{2}$ is invertible and $M_{1}$ is congruent to $M_{2}$ and $n>0$ and $M_{5}=M_{1}^{\mathrm{T}}$ and $M_{6}=M_{2}{ }^{\mathrm{T}}$, then $M_{5}$ is congruent to $M_{6}$.

(52) If $M_{4}$ is orthogonal and $M_{1}=M_{4}^{\mathrm{T}} \cdot M_{2} \cdot M_{4}$, then $M_{1}$ is similar to $M_{2}$.

Let $n$ be a natural number, let $K$ be a field, and let $M$ be a matrix over $K$ of dimension $n$. The functor $\operatorname{Trace}(M)$ yields an element of $K$ and is defined by:

(Def. 7) $\operatorname{Trace}(M)=\sum$ (the diagonal of $\left.M\right)$.

The following propositions are true:

(53) If $M_{2}=M_{1}^{\mathrm{T}}$, then $\operatorname{Trace}\left(M_{1}\right)=\operatorname{Trace}\left(M_{2}\right)$.

(54) $\operatorname{Trace}\left(M_{1}+M_{2}\right)=\operatorname{Trace}\left(M_{1}\right)+\operatorname{Trace}\left(M_{2}\right)$.

(55) $\operatorname{Trace}\left(M_{1}+M_{2}+M_{3}\right)=\operatorname{Trace}\left(M_{1}\right)+\operatorname{Trace}\left(M_{2}\right)+\operatorname{Trace}\left(M_{3}\right)$.

(56) $\operatorname{Trace}\left(\left(\begin{array}{ccc}0 & \ldots & 0 \\ \vdots & \ddots & \vdots \\ 0 & \ldots & 0\end{array}\right)_{K}^{n \times n}\right)=0_{K}$.

(57) If $n>0$, then $\operatorname{Trace}\left(-M_{1}\right)=-\operatorname{Trace}\left(M_{1}\right)$.

(58) If $n>0$, then $-\operatorname{Trace}\left(-M_{1}\right)=\operatorname{Trace}\left(M_{1}\right)$.

(59) If $n>0$, then $\operatorname{Trace}\left(M_{1}+-M_{1}\right)=0_{K}$. 
(60) If $n>0$, then $\operatorname{Trace}\left(M_{1}-M_{2}\right)=\operatorname{Trace}\left(M_{1}\right)-\operatorname{Trace}\left(M_{2}\right)$.

(61) If $n>0$, then $\operatorname{Trace}\left(\left(M_{1}-M_{2}\right)+M_{3}\right)=\left(\operatorname{Trace}\left(M_{1}\right)-\operatorname{Trace}\left(M_{2}\right)\right)+$ $\operatorname{Trace}\left(M_{3}\right)$.

(62) If $n>0$, then $\operatorname{Trace}\left(\left(M_{1}+M_{2}\right)-M_{3}\right)=\left(\operatorname{Trace}\left(M_{1}\right)+\operatorname{Trace}\left(M_{2}\right)\right)-$ $\operatorname{Trace}\left(M_{3}\right)$.

(63) If $n>0$, then $\operatorname{Trace}\left(M_{1}-M_{2}-M_{3}\right)=\operatorname{Trace}\left(M_{1}\right)-\operatorname{Trace}\left(M_{2}\right)-$ $\operatorname{Trace}\left(M_{3}\right)$.

\section{REFERENCES}

[1] Grzegorz Bancerek and Krzysztof Hryniewiecki. Segments of natural numbers and finite sequences. Formalized Mathematics, 1(1):107-114, 1990.

[2] Czesław Byliński. Functions and their basic properties. Formalized Mathematics, 1(1):5565, 1990.

[3] Czesław Byliński. Some basic properties of sets. Formalized Mathematics, 1(1):47-53, 1990.

[4] Katarzyna Jankowska. Matrices. Abelian group of matrices. Formalized Mathematics, 2(4):475-480, 1991.

[5] Katarzyna Jankowska. Transpose matrices and groups of permutations. Formalized Mathematics, 2(5):711-717, 1991.

[6] Eugeniusz Kusak, Wojciech Leończuk, and Michał Muzalewski. Abelian groups, fields and vector spaces. Formalized Mathematics, 1(2):335-342, 1990.

[7] Andrzej Trybulec. Tarski Grothendieck set theory. Formalized Mathematics, 1(1):9-11, 1990.

[8] Wojciech A. Trybulec. Groups. Formalized Mathematics, 1(5):821-827, 1990.

[9] Wojciech A. Trybulec. Vectors in real linear space. Formalized Mathematics, 1(2):291296, 1990.

[10] Xiaopeng Yue, Xiquan Liang, and Zhongpin Sun. Some properties of some special matrices. Formalized Mathematics, 13(4):541-547, 2005.

[11] Katarzyna Zawadzka. The product and the determinant of matrices with entries in a field. Formalized Mathematics, 4(1):1-8, 1993.

Received January 4, 2006 